\title{
HEATING OF THE QUIET SOLAR CHROMOSPHERE
}

\author{
I. Role of the Inner Network Bright Points
}

\author{
R. KARIYAPPA, K. R. SIVARAMAN \\ Indian Institute of Astrophysics, Bangalore-560034, India \\ and \\ M. N. ANADARAM \\ Department of Physics, Bangalore University, Bangalore-560056, India
}

(Received 26 September, 1992; in revised form 2 February, 1994)

\begin{abstract}
We have analyzed a large number of $\mathrm{Ca}$ II $\mathrm{H}$ line profiles at the sites of the bright points in the interior of the network using a 35-min-long time sequence of spectra obtained at the Vacuum Tower Telescope (VTT) of the Sacramento Peak Observatory on a quiet regon of the solar disc and studied the dynamical processes associated with these structures. Our analysis shows that the profiles can be grouped into three classes in terms of their evolutionary behaviour. It is surmized that the differences in their behaviour are directly linked with the inner network photospheric magnetic points to which they have been observed to bear a spatial correspondence. The light curves of these bright points give the impression that the 'main pulse', which is the upward propagating disturbance carrying energy, throws the medium within the bright point into a resonant mode of oscillation that is seen as the follower pulses. The main pulse as well as the follower pulses have identical periods of intensity oscillations, with a mean value around $190 \pm 20 \mathrm{~s}$. We show that the energy transported by these main pulses at the sites of the bright points over the entire visible solar surface can account for a substantial fraction of the radiative loss from the quiet chromosphere, according to current models.
\end{abstract}

\section{Introduction}

One of the major problems in solar physics is to understand the way the chromosphere is heated and supported by the non-radiative energy from the layers below. With the revival of interest (Anderson and Athay, 1989; Kalkofen, 1989) in using the long-period acoustic waves in theoretical modelling as the agent for the heating of the quiet chromosphere, a corresponding need for re-examining the contributions from the quiet chromospheric structures to the required energy flux has arisen. The recent elaborate calculations of Anderson and Athay (1989) have led to a fairly precise estimate of the energy radiated by the Fe II, $\mathrm{Mg}$ II, Ca II ions and other species, and this is far in excess of the values given by the often quoted model $\mathrm{C}$ of VAL81 (Vernazza, Avrett, and Loeser, 1981). This acts as a further impetus to re-examine the dynamical process of the structures in the quiet chromosphere and to find out from the observational evidence how the energy requirements can be met.

The chromosphere appears highly structured on a two-dimensional image of the Sun in the $\mathrm{H}$ or $\mathrm{K}$ line of $\mathrm{Ca}$ II. The inhomogeneities seen in emission are the plages, the network and the bright points (with dimension 1-2 arc sec) that populate the interior of the network. The bright cell points (Bappu and Sivaraman, 
1971; Liu, 1974; Zirin, 1974; Cram and Dame, 1983) seen with high contrast in spectroheliograms taken with a narrow passband isolating the $K_{2 \mathrm{~V}}$ emission peak (Hale and Ellerman, 1903) are also referred to as the $K_{2}$ grains or cell grains (Beckers, 1964; Rutten and Uitenbroek, 1991). We use the term bright points in this paper. In addition, within the network there are regions in weak emission between the bright points forming the unresolved 'truly quiet chromosphere' and the dark condensations which are columns of absorbing matter (Bappu and Sivaraman, 1971). Among these chromospheric inhomogeneities, the network, the bright points and the regions in betweem them characterize the quiet solar chromosphere, and the emission features that stand out prominently are the bright points, besides the network.

The sequence of events associated with the upward propagation of the brightness disturbance at the site of the bright points when seen in the $\mathrm{H}$ or $\mathrm{K}$ line is known from earlier investigations (Punetha, 1974; Liu, 1974; Cram, 1974; Cram and Dame, 1983; and Dame, 1984). Briefly, the passage of this disturbance starting from the ambient level grows into an intense brightening of the bright point and subsides to the ambient level. This cycle lasts for $\sim 200 \mathrm{~s}$. This is followed by many such disturbances almost of the same period. This periodic brightening has been identified with the $\sim 3$-min chromospheric oscillations noticed by Jensen and Orall (1963). The detailed analysis of the evolution of the H-line profiles by Cram and Dame (1983) provides many more examples of events such as those detected by Liu (1974) and also differentiates between the evolution of the $\mathrm{H}$-line profiles at the site of the bright points and the network boundaries. A good deal of theoretical work in modelling these fine structures and the waves that can propagate in them also exists. The recent review by Rutten and Uitenbroek (1991) gives a comprehensive account of the current status in our knowledge both in observational and theoretical fronts on the $K_{2 V}$ (and $H_{2 V}$ ) bright points. Considering the importance of these structures, the amount of observational results that can serve as useful inputs for the construction of theoretical models are rather meagre and leave much to be desired. Some of the important questions which remain unanswered are:

(i) Do all bright points behave in the same way when the propagating disturbances pass through them?

(ii) What is the role of the magnetic fields in this dynamical process?

(iii) Do the brightness enhancements occur in a random fashion or do they show any spatial and temporal coherence in their behaviour?

(iv) What is the physical nature of the pulse and the nature of the wave that carries the energy and dissipate at the chromospheric level?

In this paper we concentrate on the study of the time evolution of the bright points from an examination of their $\mathrm{H}$-line profiles and attempt to provide answers to some of the questions raised above. 


\section{Observations}

The data for this study consist of a photographic time sequence of spectra in the H-line region obtained at the Vacuum Tower Telescope (VTT) and the echelle spectrograph of the Sacramento Peak Observatory, on September 13, 1971 by Jacques Beckers and Brown based on the observing propasal by one of us (K.R.S.) and J. Beckers. The image has a scale of $3.48 \mathrm{arc} \mathrm{sec} \mathrm{mm}^{-1}$. The entrance slit with a width of $144 \mu$ (or 0.5 arc sec) was positioned in the north-south direction on the sky and was located on a quiet region around the centre of the solar disc. Its length of $80 \mathrm{~mm}$ covered $280 \mathrm{arc} \mathrm{sec}$ on the Sun. The scheme of observations used program B of the HIRKHAD mode (Beckers et al., 1972) which takes spectra simultaneously in seven lines at a repetition rate of $12 \mathrm{~s}$. The solar rotation was compensated for during the observations. The exposure for each frame was $\sim 3 \mathrm{~s}$ and the spectra were obtained on Kodak 5375 emulsion in $70 \mathrm{~mm}$ format at a dispersion of $12.1 \mathrm{~mm} \mathrm{~A}^{-1}$ (15th order) in the H-line region. The seeing conditions were exceptionally good during the entire duration of $35 \mathrm{~min}$. With $12 \mathrm{~s}$ for each frame we have in all 177 frames. The present study deals with the analysis of only the $\mathrm{H}$ line.

\section{Reduction of the Data}

In Figure 1 we have shown one frame from this sequence. We have chosen 32 locations for a detailed study and have designated them as $B_{1}, B_{2}, B_{3}, \ldots B_{32}$ (Figure 1). The images of two hair lines were used as the reference fiducial marks to fix these 32 locations. Of these, 29 locations are in bright points and 3 locations $\left(B_{4}, B_{18}, B_{26}\right)$ are in network boundaries. This paper deals only with the study of the bright points. A visual examination of the frames showed that some bright points were strikingly bright whereas some others never reached this level of brightness in the entire duration of the sequence. We therefore chose these 29 bright points so as to have enough points for analysis from the two categories without any bias. The identity of all the 29 bright points was confirmed through slit jaw pictures taken in the $\mathrm{K}$ line. We obtained the digital values of the densities for these 29 locations in all the frames by scanning parallel to the dispersion with the PDS microphotometer with a projected size of $50 \mu \times 200 \mu$ for the scanning aperture which corresponds to $0.004132 \AA \times 500 \mathrm{~km}$. We converted the density values first to relative intensity via a photometric calibration taking into account the stray light and then in terms of the continuum intensity by setting the intensity at $\lambda 3966.25 \AA$ on the violet wing of the $\mathrm{H}$ line as $23 \%$ of the continuum (White and Suemoto, 1968). The mean of the quiet profiles showed very close agreement with the photoelectric profile of White and Suemoto (1968). Thus we have in all 5133 photometrically calibrated profiles for these 29 bright point locations. We derived for each profile the following five parameters and made plots of these versus time covering the duration of the sequence:

(i) $I_{H 2 V}$, intensity of the emission peak in the violet; 


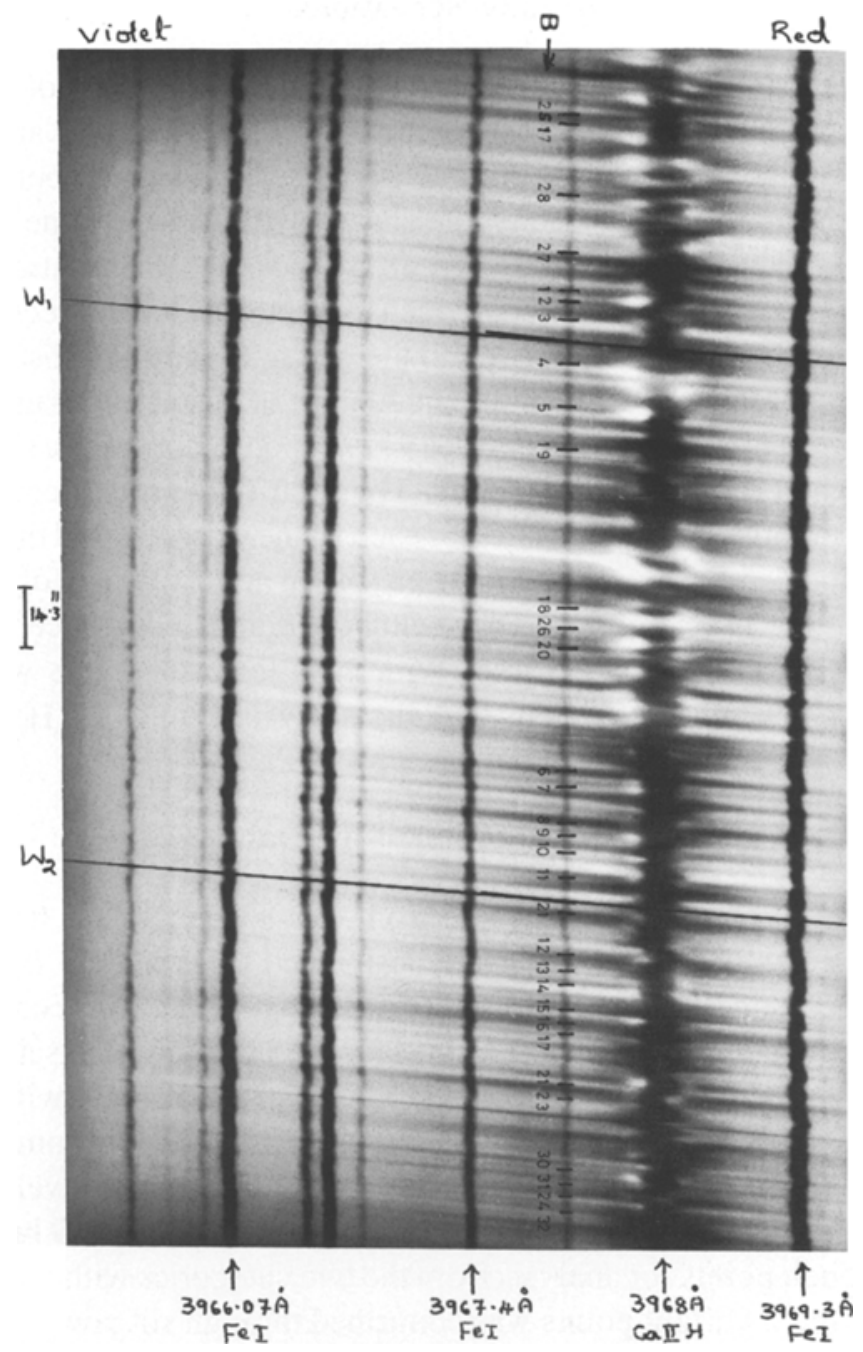

Fig. 1. Enlarged photographic print of frame No. 209 of the Ca II H spectra from the 35-min-long sequence. $W_{1}$ and $W_{2}$ are the images of the two hair lines used as the local reference for positions. The 32 features $B_{1}, B_{2}, \ldots, B_{32}$ are marked for identification.

(ii) $I_{H 2 R}$, intensity of the emission peak in the red;

(iii) $I_{H 3}$, intensity of the $\mathrm{H}_{3}$ absorption core;

(iv) $I_{H 2 V} / I_{H 2 R}$, the ratio of the intensities; and

(v) $\Delta \lambda_{H 3}$, the Doppler shift of the $H_{3}$ core. 


\section{Results and Discussions}

\subsection{TIME-DEPENDENT PROPERTIES OF THE PROFILES OF THE BRIGHT POINTS}

Although at first sight our $\mathrm{H}$-line profiles of the bright points seem amazingly diverse in their forms during evolution, we find that they can broadly be grouped into three classes. Because of this diversity, the term 'typical evolution of a brightpoint profile' (Liu, 1974; Durrant, Grossman-Doerth, and Kneer, 1976) has only a restricted sense representing just one class of profiles.

Class I, the bright points in this class show a large enhancement of $I_{H 2 V}$ at their peak brightness phase, as high as 3 times above the mean ambient level (Figures 2 and 3), which corresponds to the undisturbed line profile (at time $t=0$ ) of Figure 11. Of the 12 samples in this class, we show the light curves of $B_{17}, B_{19}$, $B_{22}$, and $B_{26}$ in Figure 2 and of $B_{1}, B_{2}$, and $B_{5}$ in Figure 3 . The light curves of the remaining 5 bright points are similar to these.

Class II, bright points show moderate intensity enhancement in $I_{H 2 V}$ (about twice the mean ambient level) at the peak brightness phase. Of the 10 samples, we show in Figure 4 the light curves of $B_{12}, B_{13}$, and $B_{14}$ to represent this class.

Class III, bright points show only a marginal increase in $I_{H 2 V}$ at the brightest phase. Of the 7 samples, we show in Figure 5 the light curves of $B_{8}, B_{9}, B_{10}$, and $B_{11}$.

\subsubsection{Properties of Evolution of the Profiles in the 3 Classes}

(a) Number and amplitude of the wave pulses. For the class I bright points (e.g., $B_{17}$ and $B_{19}$ in Figure 2) we designate the highest peak of each bright point as the 'main pulse' (we use the term pulse in the ordinary sense to bring out the rapid rise and fall in intensity) and mark it as $P_{1}$. The $\mathrm{H}$-line profile corresponding to the peak $P_{1}$, has $I_{H 2 V} / I_{H 2 R} \sim 2.0$ or higher, and we have used this value for the ratio as an objective criterion to pick out $P_{1}$ from the plots. For $B_{12}$ (class II) the main pulse $P_{1}$ occurred $1.7 \mathrm{~min}$ after the start of the observations and its amplitude is much smaller than that in class I. In class II, the main pulse for $B_{9}$ (Figure 1) is weaker than that in $B_{12}$. To establish the reality of the three classes, we have picked out all the peaks $\left(P_{1}\right)$ from the $H_{H 2 V}$ vs time plots of the 29 bright points. In class I there is only one $P_{1}$ in each plot, whereas in classes II and III there is more than one peak. We have made a peak size histogram from these peak values (Figure 6). The peaks fall into three groups showing that the classification is valid.

Although our sample of profiles is large we do not have even a single case in class I where more than one $P_{1}$ has occurred during the $35-\mathrm{min}$ observation. These are photographic spectra, and the limitation on the duration was imposed by the total length of one roll of film manufactured by Kodak Co. We propose to acquire sequences lasting 2 to 3 hours to see how often the tall peaks $\left(P_{1}\right)$ occur in class I bright points and whether they are periodic or purely random. Meanwhile we examined the $I_{K 2 \mathrm{~V}}$ plot (Figure 2 of Kulaczewski, 1992) drawn from a sequence lasting 80 min which shows that the tall peaks occur randomly. Since his plot is for an 


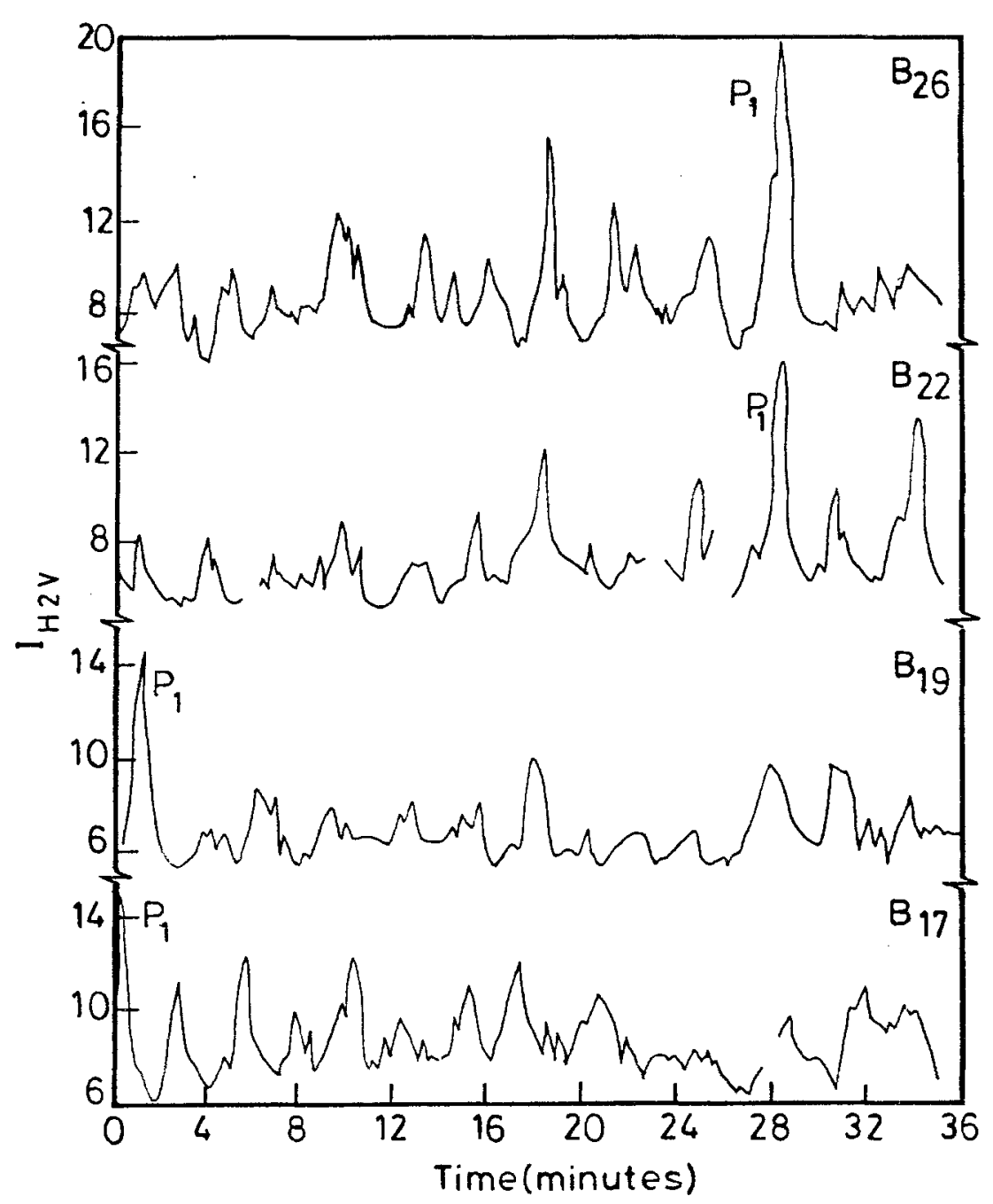

Fig. 2. The variations in intensity of the $H_{2 V}$ emission peak ( $\left.I_{H 2 V}\right)$ of the four bright points $\left(B_{17}\right.$, $B_{19}, B_{22}$, and $B_{26}$ ) during the 35 -min observations. These samples belong to class $I$. The main impulse designated as $P_{1}$ is 4 to 5 times the normal brightness value and is followed by several pulses with decreasing amplitudes.

arbitrary cell interior position, it does not presumably represent a typical energetic bright point - like $B_{1}$ or $B_{5}$. For $B_{17}$ and $B_{19}$ a minimum of 8 to 10 pulses (this number could even be more) follow the main pulse and this seems to be a common feature of the bright points of class I. For those of class II and class III 4-6 follower pulses seem most common although there are exceptions. In $B_{11}$ and $B_{12}$ there are about 7 follower pulses. In $B_{8}$, most of the pulses have the same amplitude and so $P_{1}$ is not easily identifiable. $B_{1}$ and $B_{17}$ indicate that the amplitudes of the follower pulses decay with time. On the other hand, the plots for $B_{5}, B_{26}$, would give an 


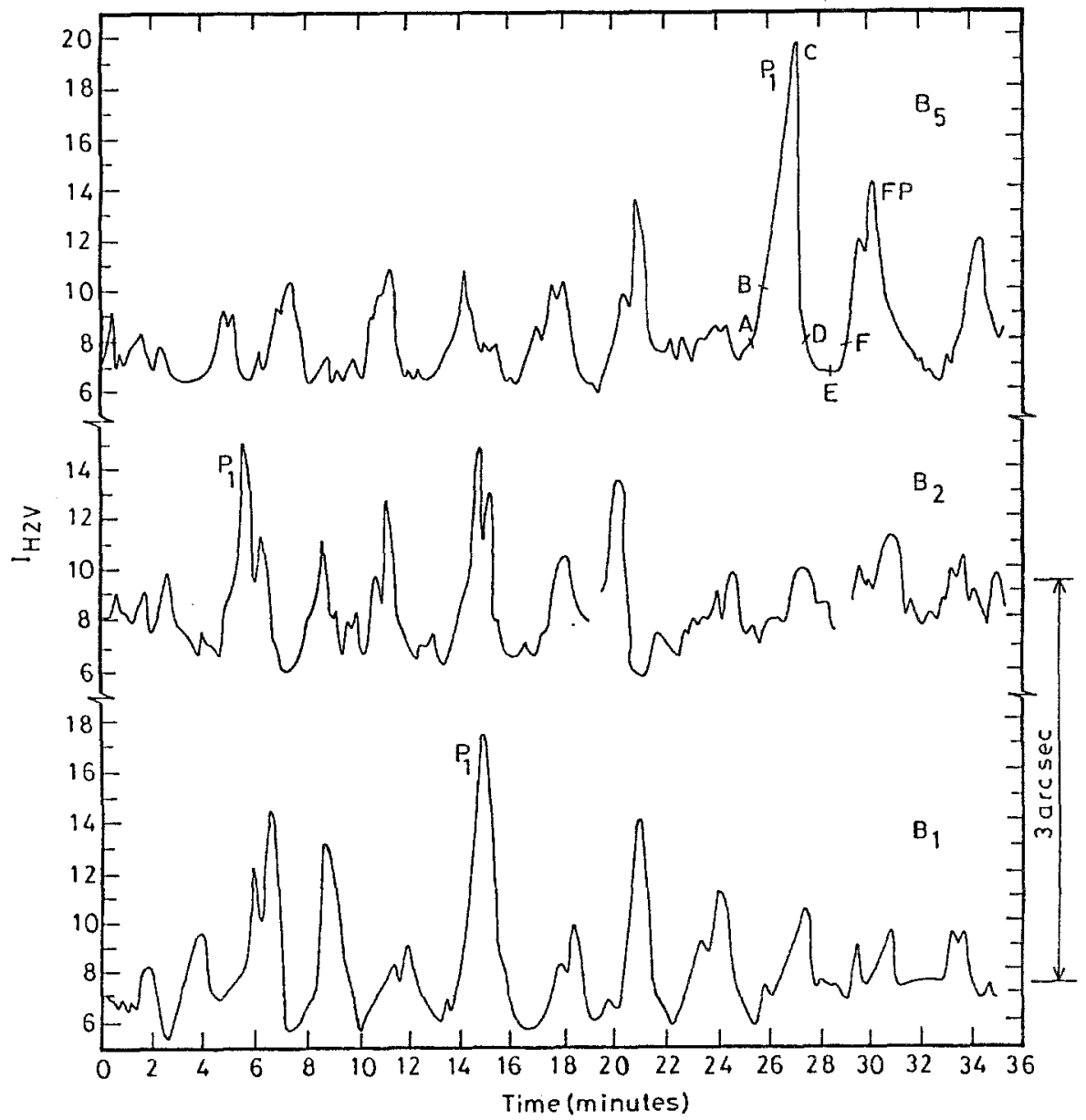

Fig. 3. Additional samples of light curves of bright points ( $B_{1}, B_{2}$, and $B_{5}$ ) of class I. $P_{1}$ represents the main pulse and FP the follower pulse.

impression that one can as well draw curves towards the highest peak showing an exponential growth of the amplitudes towards the highest peak. Deubner (1991) suggests that these peaks simply portray wave interference without carrying much information. Although there are variations as discussed above we suggest the possibility that the main pulse is followed by several (8-10 or more) pulses whose amplitudes decay with time, and this pattern is more or less maintained for all the three classes in our data. However, a longer sequence is desirable to confirm this. We have fitted exponential functions for $B_{1}, B_{5}, B_{17}, B_{19}, B_{27}, B_{31}$ (class I; Figure 7); for $B_{15}$ and $B_{21}$ (class II) and for $B_{9}$ and $B_{11}$ (class III) and these are presented in Table I. The slopes are more or less the same, and one value of the 


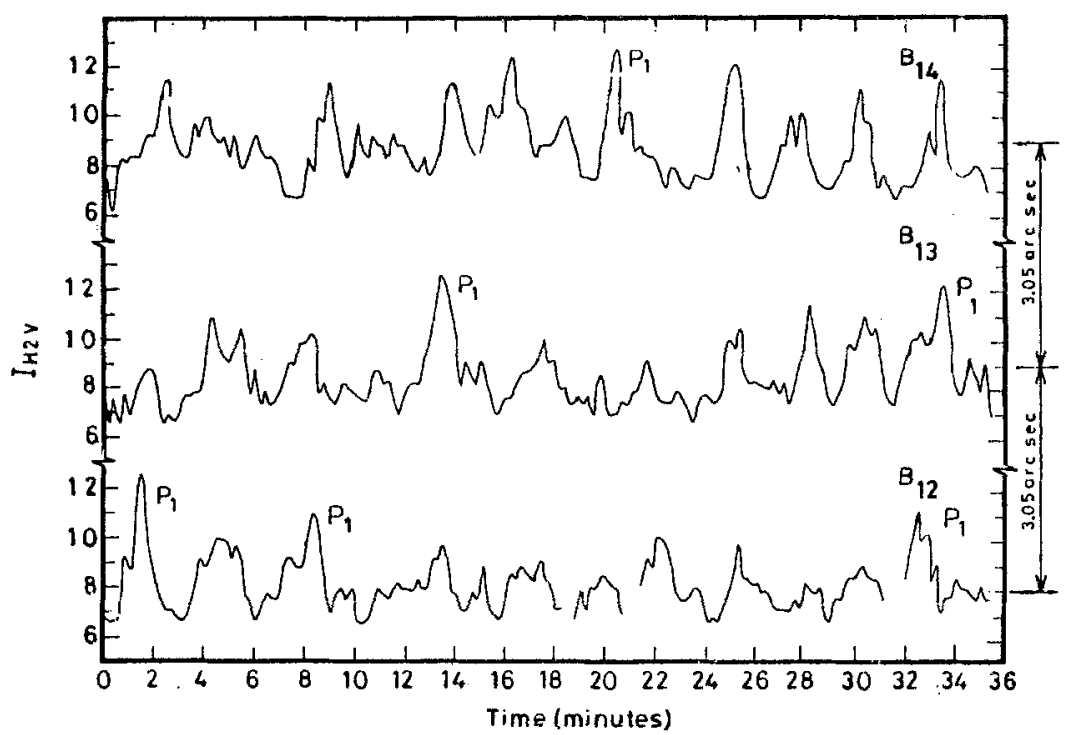

Fig. 4. Light curves of the bright points $B_{12}, B_{13}$, and $B_{14}$. The main pulse ( $\left.P_{1}\right)$ is only 2 to 3 times the normal value of brightness. These belong to class II.

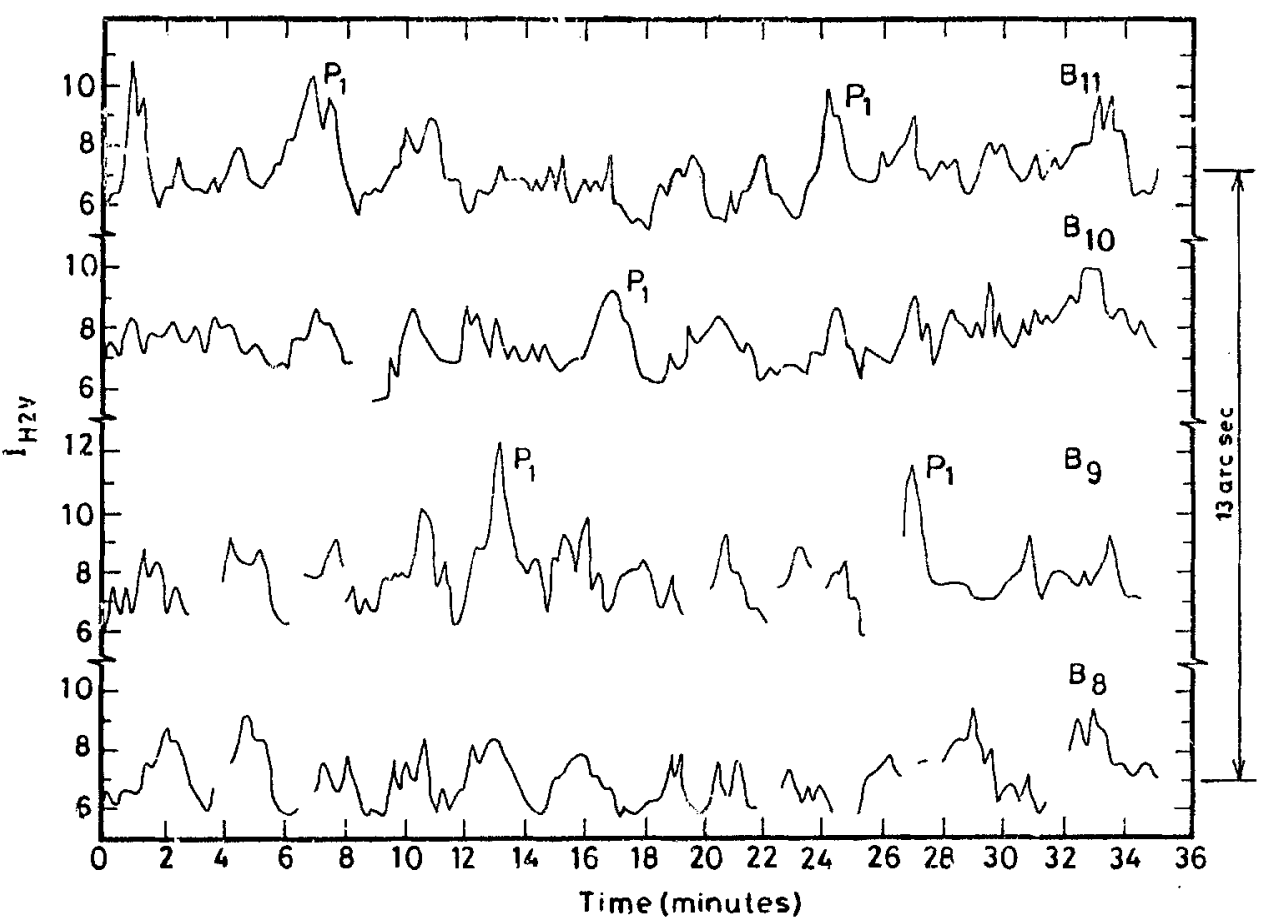

Fig. 5. Light curves of the bright points $B_{8}, B_{9}, B_{10}$, and $B_{11}$. The main pulse $\left(P_{1}\right)$ is only 1.1 to 2.0 times the normal value of brightness. These belong to class III. 


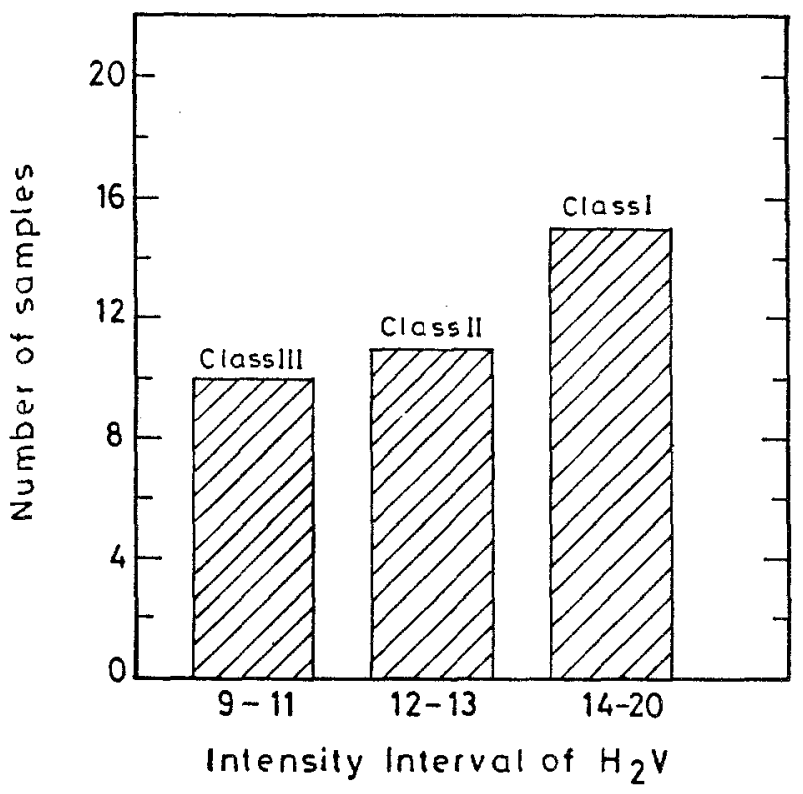

Fig. 6. Histogram showing the distribution of the intensity of the main pulse $P_{1}$ of the 3 classes.

exponent $\left(4.14 \times 10^{-4}\right)$ can represent reasonably well the decay for all the cases. Liu's (1974) plots (his Figures 6(b) and 6(c)) also show the main pulse and the exponential decay of the follower pulses and a similar fit done by us to his data gives $3.6 \times 10^{-4}$ for the exponent which is comparable to our values presented in Table I.

(b) Period of oscillations. The main pulse as well as most of the follower pulses, although periodic, show a rapid rise and fall in brightness suggesting a nonlinear behaviour. We have evaluated the period of the pulses constituting a wave train by feeding the $I_{H 2 V}$ digital values (values every $12 \mathrm{~s}$, corresponding to the repetition rate of the frames) to a computer and have measured the time separation between consecutive peaks. The histogram of the periods so derived for the main pulses as well as for the follower pulses for the 29 bright points show that the periods of these brightness oscillations lie in the range 150-210 s with the peak in the bin $190 \pm 20 \mathrm{~s}$ (Figure 8). We have also done a power spectrum analysis for all the bright points and these are shown in Figures 9 and 10. Although there are secondary peaks in some cases, one noteworthy point is that the average period falls in the range $190 \pm 20 \mathrm{~s}$. The period is almost the same for the pulses in the three classes and thus seems to be independent of the differences in the brightness enhancement and within a class the main pulse and the follower pulses have also the same period. From these considerations we propose that the main pulse is the perturbing disturbance which throws the medium within the bright point into a resonant, oscillating mode seen as the follower pulses with the same period as the 


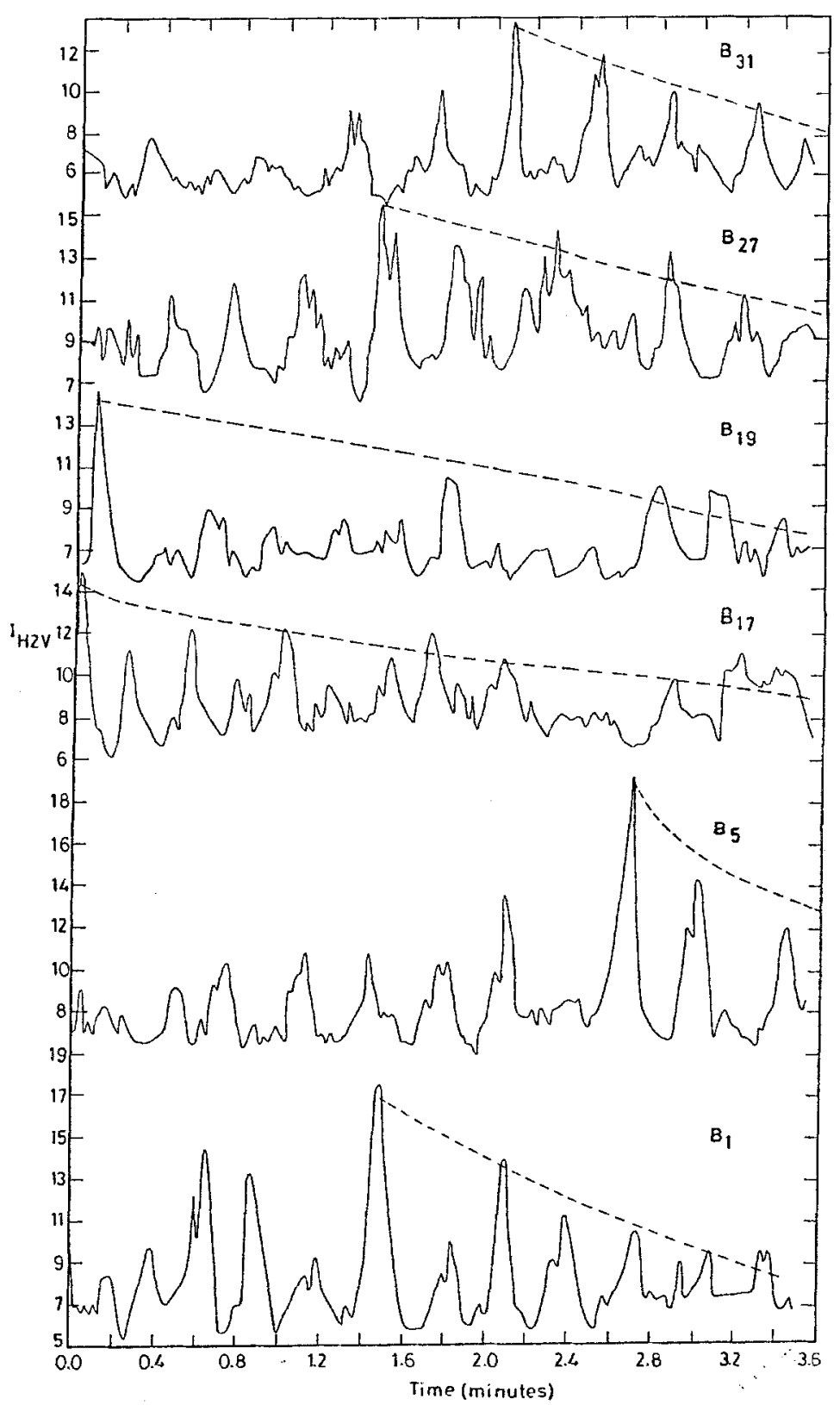

Fig. 7. Exponential function fitted to the brightness decay in the amplitudes of the follower pulses for the bright points $B_{1}, B_{5}, B_{17}, B_{19}, B_{27}$, and $B_{31}$. The values of the exponential fit can be seen in Table I. The slopes are more or less the same.

exciting pulse and decaying exponentially.

In order to relate the dynamical evolution of the profiles with the changes occurring at the site of a bright point, we have traced the sequence of events in one 

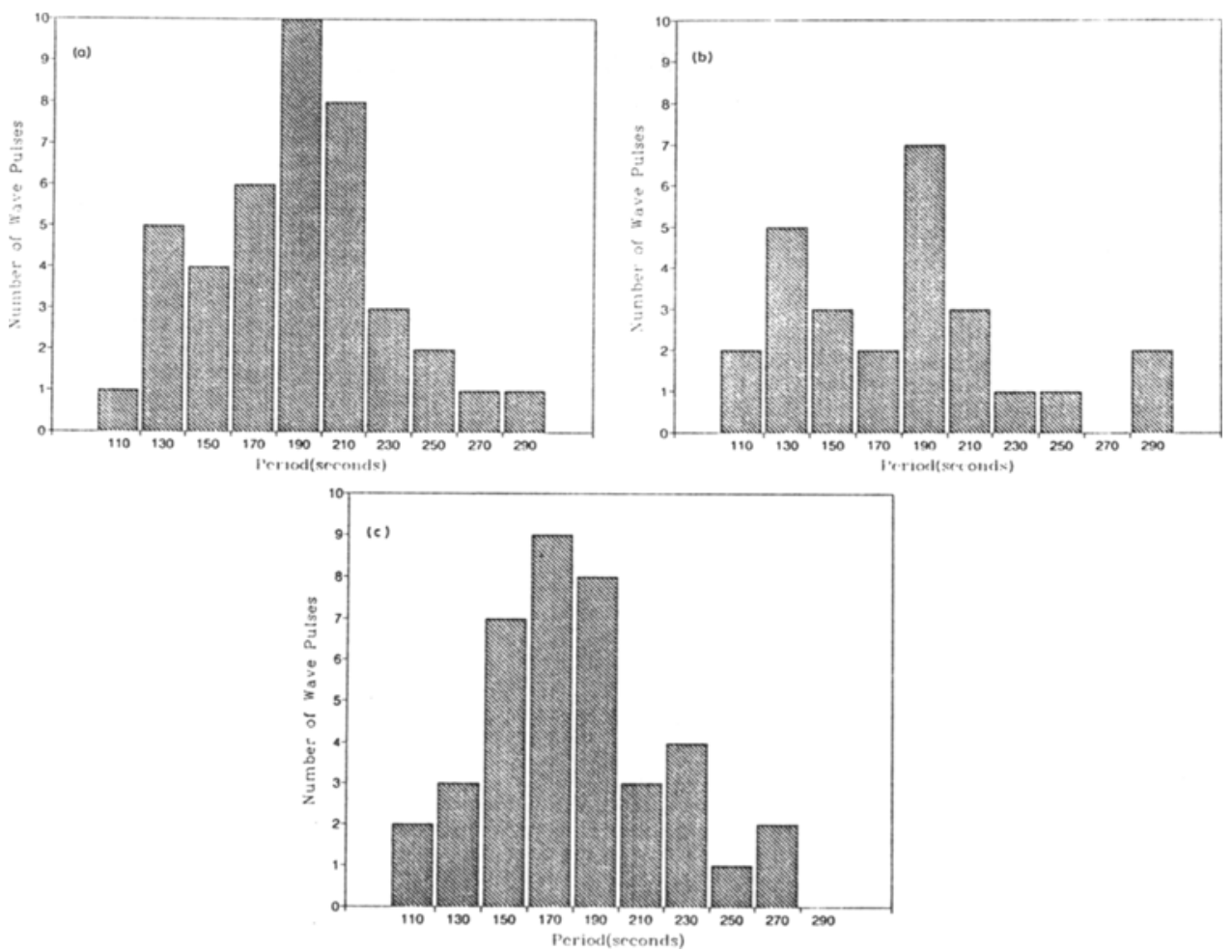

Fig. 8. Histogram plots of the periodicity of oscillations in $I_{H 2 \mathrm{~V}}$ for the three classes of bright points. (a) Class I, (b) class II, (c) class III. There is a sharp peak at $190 \mathrm{~s}$ in class I and II and $170 \mathrm{~s}$ in class III bright points.

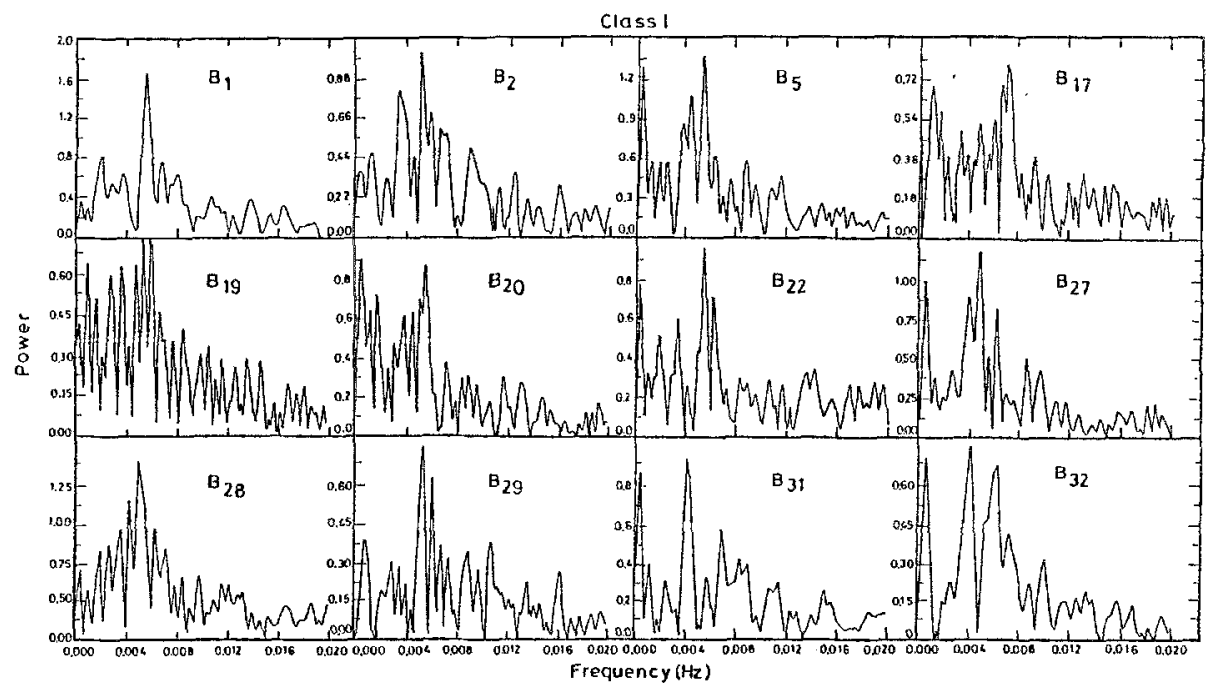

Fig. 9. Power spectra of 12 bright points of class I. Notice the main peak is invariably centred around $190 \pm 20 \mathrm{~s}$. 
TABLE I

Exponential decay in the amplitudes of the follower pulses

\begin{tabular}{lll}
\hline Class & Bright point & $\begin{array}{l}\text { Exponential function } \\
I(t)\end{array}$ \\
\hline I & $B_{1}$ & $16.69 \exp \left(-5.9 \times 10^{-4} t\right)$ \\
& $B_{5}$ & $18.89 \exp \left(-4.9 \times 10^{-4} t\right)$ \\
& $B_{17}$ & $14.63 \exp \left(-3.2 \times 10^{-4} t\right)$ \\
& $B_{19}$ & $13.66 \exp \left(-3.7 \times 10^{-4} t\right)$ \\
& $B_{22}$ & $15.21 \exp \left(-4.6 \times 10^{-4} t\right)$ \\
& $B_{27}$ & $15.84 \exp \left(-3.3 \times 10^{-4} t\right)$ \\
& $B_{28}$ & $19.87 \exp \left(-3.5 \times 10^{-4} t\right)$ \\
& $B_{31}$ & $13.58 \exp \left(-4.9 \times 10^{-4} t\right)$ \\
II & $B_{12}$ & $11.78 \exp \left(-3.2 \times 10^{-4} t\right)$ \\
& $B_{15}$ & $11.48 \exp \left(-5.6 \times 10^{-4} t\right)$ \\
& $B_{21}$ & $12.44 \exp \left(-2.4 \times 10^{-4} t\right)$ \\
III & $B_{9}$ & $12.20 \exp \left(-4.7 \times 10^{-4} t\right)$ \\
& $B_{11}$ & $10.90 \exp \left(-3.9 \times 10^{-4} t\right)$ \\
\hline
\end{tabular}

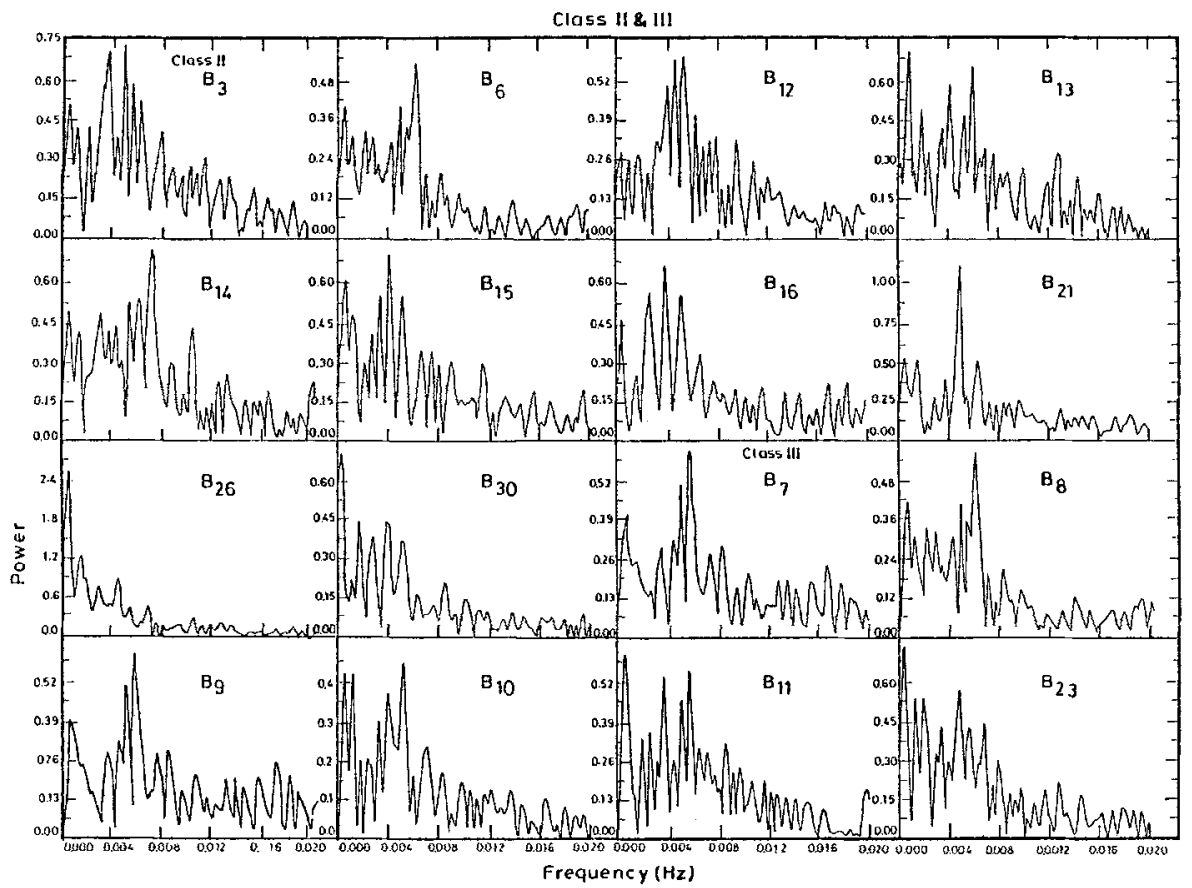

Fig. 10. Same as Figure 9 for the bright points of class II $\left(B_{3}, B_{6}, \ldots, B_{30}\right)$ and of class III $\left(B_{7}, B_{8}\right.$, $\ldots, B_{23}$ ). 


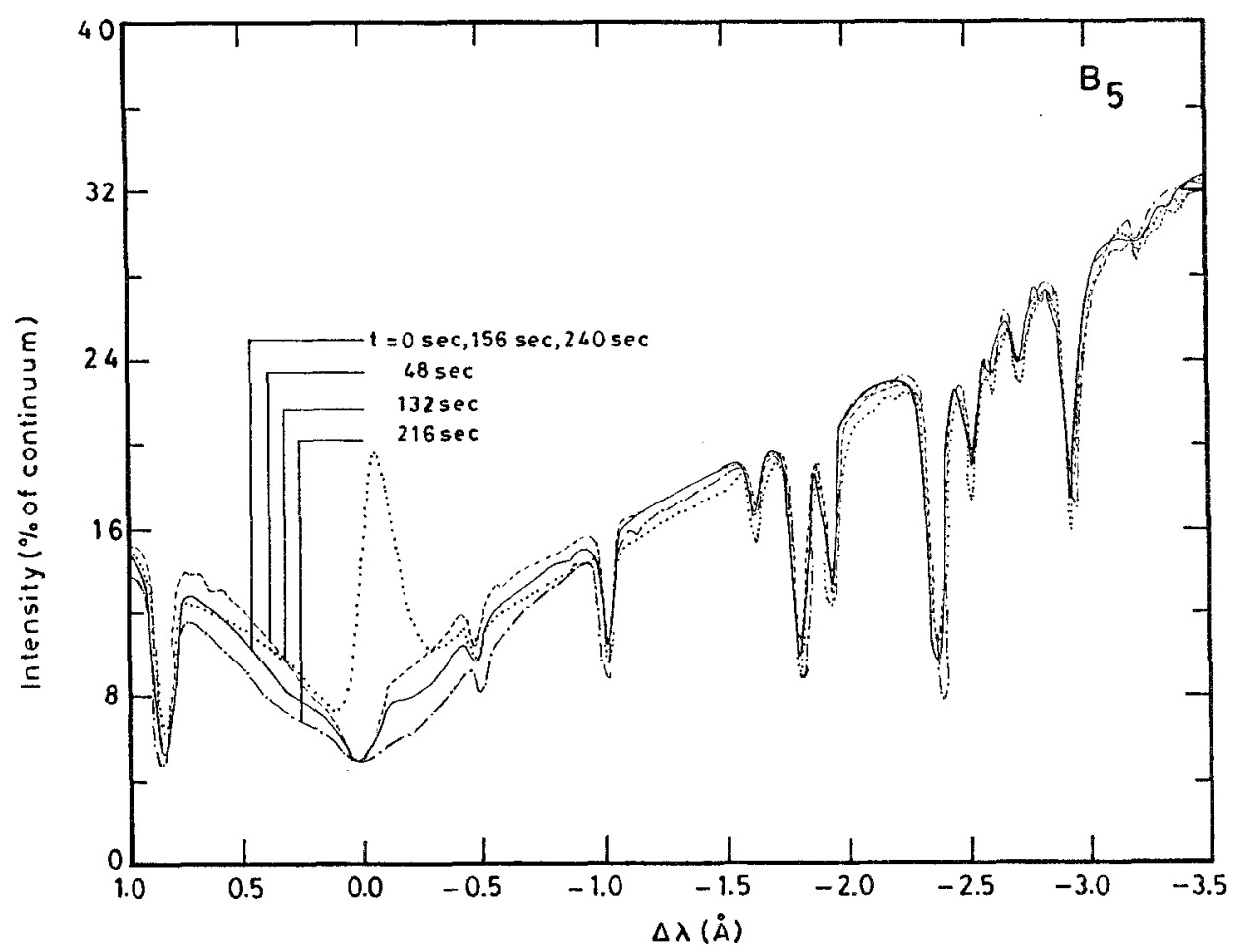

Fig. 11. Dynamical evolution of the H-line profile of class I bright point $B_{5}$. See text for details.

full cycle during the passage of the main pulse, $P_{1}$, by picking out the line profiles corresponding to 6 specific locations on the $H_{2 V}$ light curve of the bright point $B_{5}$ (A to $\mathrm{F}$ on the main pulse $P_{1}$ of bright point $B_{5}$ in Figure 3). The reader is requested to refer to Figures 3 and 11 alternately to follow the events. The profile at $t=0$ (Figure 11) represents the undisturbed condition (corresponding to location A on $P_{1}$, Figure 3) with $I_{H 2 V}$ and $I_{H 2 R}$ approximately equal and serves as the reference profile. At $t=48 \mathrm{~s}$ (position $\mathrm{B}$ on $P_{1}$ ) the brightenings have already reached symmetrically on both wings. At $t=132 \mathrm{~s}$ (position $\mathrm{C}$ ) the brightening has propagated to the $\mathrm{H}_{2}$ level and this is seen as an increase in $I_{H 2 \mathrm{~V}}$ by a factor of 3 and in some cases even as much as by a factor of 5 or 6 . Simultaneously $\mathrm{H}_{3}$ shows a large red shift. At $t=156 \mathrm{~s}$ (position $\mathrm{D}$ which is a mirror image of position A in $P_{1}$ ) the profile looks similar to that of $t=0$, marking the culmination of the passage of the brightness disturbance of $P_{1}$ and thus of the brightening phase. Now onwards is the dark whisker phase. At $t=216 \mathrm{~s}$ (position $\mathrm{E}$ ) it has the shape of a pure absorption profile and this corresponds to the dark whisker on the spectrum. In the last stage of the dark whisker phase ( $t=240 \mathrm{~s}$, position F) the bright threads have already made their appearance on either wing, whereas the core of the $\mathrm{H}$ line is yet to recover from the dark whisker phase and so presents the shape of a pure absorption profile. It is now clear that the profile of this shape occurs routinely at 
the beginning of every cycle as part of the evolutionary course. This was noticed by Liu (1974). The bright threads soon propagate towards the $\mathrm{H}$ line and the whole cycle of events repeats for the follower pulse FP (Figure 3), but less dramatically.

In our example, the duration of one complete cycle is $\sim 240 \mathrm{~s}$; it could be even less by one frame ( $\sim 228 \mathrm{~s}$ ) or two, but our time resolution of $12 \mathrm{~s}$ does not allow a better estimation. Although the duration varies between the extreme values of 2 and 5 min (Rutten and Uitenbroek, 1991), there are many examples in $B_{5}$ itself where the full cycled takes only 180-200 s, and thus the period lies in the neighbourhood of $190 \mathrm{~s}$. The profiles of bright points in class II and class III also exhibit similar evolution during the passage of the main pulse but with reduced brightness enhancements and therefore is not as picturesque as those of class I. It is now clear that the dynamical evolution observed and described by Punetha (1974), Liu (1974), and Cram and Dame (1983) all pertain to the bright points of class I.

The main pulse, when plotted on a large scale, shows a sawtooth shape with a slow rise time to maximum brightness $(\sim 120 \mathrm{~s})$ and a fast falling time $(\sim 35 \mathrm{~s})$, although this becomes less apparent after a few pulses. The follower pulses also tend to show a sawtooth shape. This is in contrast to the fast (10 s) rise to maximum brightness followed by a slow decay (100 s) noticed by Cram and Dame (1983). The main pulses of class II and III, which are weaker than those of class I, do not show this shape; even in class I the sawtooth shape becomes measurable only when the main pulse has a large increase in intensity (e.g., $B_{1}$ and $B_{5}$ ).

(c) Doppler motions. We find that as $I_{H 2 V}$ brightens during the passage of the main pulse, the core of $\mathrm{H}_{3}$ shows a fast and systematically increasing redward shift $\left(\Delta \lambda_{H 3}\right)$, attaining a maximum value simultaneously with the maximum brightness phase of $H_{2 V}$ emission (Figure 12). These are referred to as the $H_{2 V}$ flash and $H_{3}$ Doppler manoeuver by Rutten and Uitenbroek (1991). This redward shift in $H_{3}$ completely masks the $H_{2 R}$ emission resulting in a highly unsymmetric profile. This Doppler motion quickly swings back to the normal wavelength position with the fall in $I_{H 2 V}$ resembling a sawtooth pattern. The $H_{2 V}$ emission peak itself exhibits a redward Doppler shift that mimics the shift $\Delta \lambda_{H 3}$, although it is smaller. The maximum value of the red shifts in $H_{3}\left(\Delta \lambda_{3}\right)$ and $H_{2 V}\left(\Delta \lambda_{H 2 V}\right)$ are $7.7 \mathrm{~km} \mathrm{~s}^{-1}$ and $5.3 \mathrm{~km} \mathrm{~s}^{-1}$, respectively, whereas $H_{1 V}$ absorption shows a large violet shift $\left(9.5 \mathrm{~km} \mathrm{~s}^{-1}\right)$ during the passage of the pulse. These wavelength shifts associated with the passage of every main pulse appear to be a regular part of the evolutionary phenomenon. From Figure 12 one gets the impression that the $\mathrm{H}$-line forming layers are compressed by the approaching layers $H_{3}, H_{2}$, and $H_{1}$. This would enhance the temperature and alter the opacity significantly over the ambient values. The same pattern of events also occurs during the passage of the main pulse in the class II and class III bright points (Figure 12). However these are far less distinct.

What can cause the differences in behaviour of the bright points in the three classes? Sivaraman and Livingston (1982) have shown that the bright points bear a one-to-one spatial correspondence with the arc sec inner network magnetic ele- 


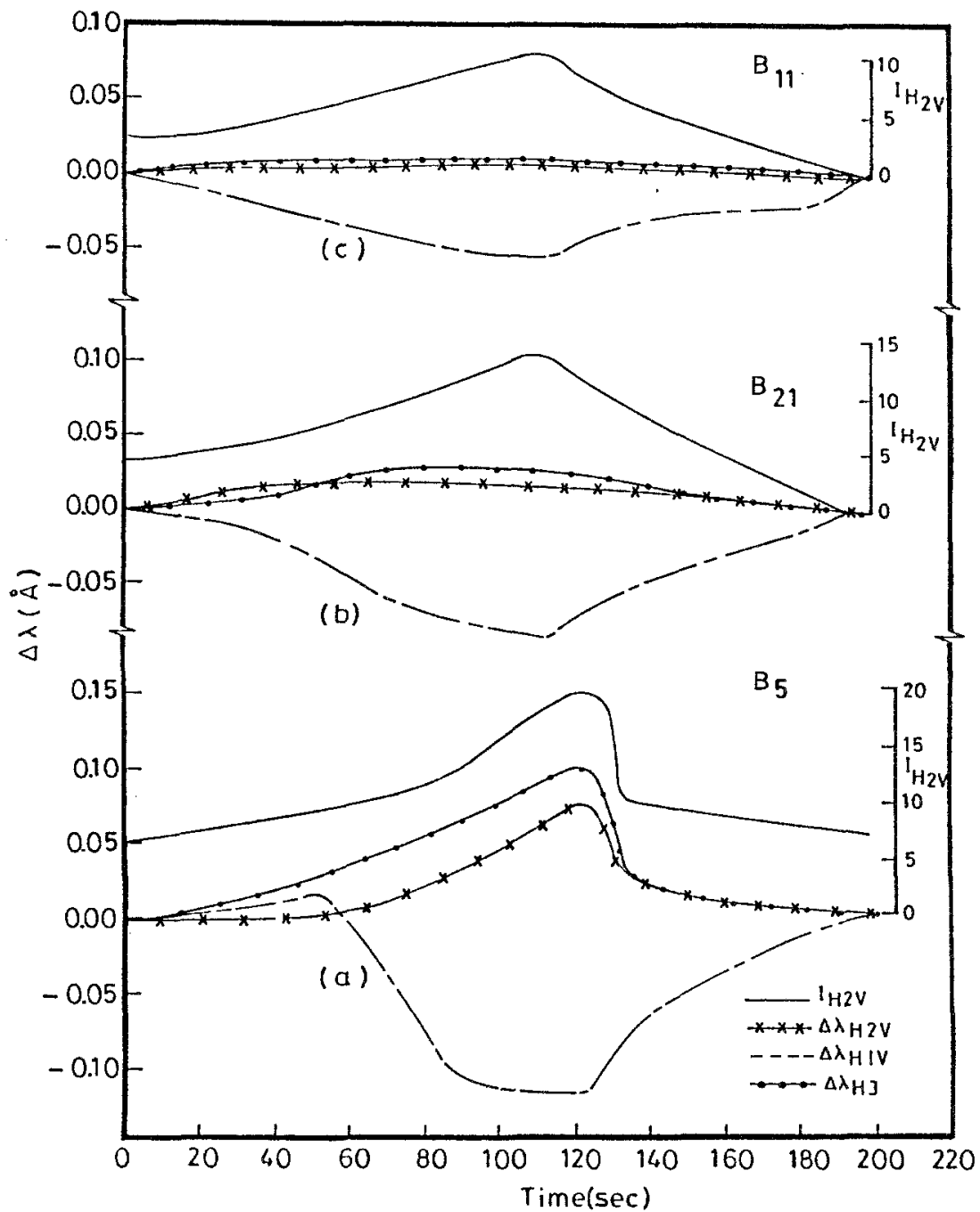

Fig. 12. Doppler motions in the wavelengths positions of $H_{3}, H_{2 V}$, and $H_{1 V}$ during the passage of the main pulse for the three classes of bright points. (a) Class I $\left(B_{5}\right)$, (b) class II $\left(B_{21}\right)$, and (c) class III ( $B_{11}$ ). The redward displacements in $H_{3}$ and $H_{2} \mathrm{~V}$ are simultaneous with the violetward displacement in $H_{1 V}$.

ments at the photospheric level. In addition, they find that the strong $H_{2 V}$ bright points correspond to higher field strength locations. We surmize from their observations that the bright points of class I would spatially correspond to those locations of inner network elements with the highest fields they have observed, and those of class III with locations of the weakest fields.

Dame (1984) has prepared two-dimensional frequency maps from equispaced intensity traces (his Figure 5) which show that the oscillatory power is exclusively concentrated in well-defined, discrete points, namely the bright points. From this, 
he suggests that the oscillating cell point locations cannot be random (as in 3dimensional interference of waves) but would imply the connection with a fixed underlying structure like the magnetic point.

\subsection{THE SPATIAL COHERENCE IN THE BEHAVIOUR OF THE BRIGHT POINTS}

The plots of $I_{H 2 V}$ versus time (Figures 3, 4, and 5) suggest that the intensity oscillations in adjacent bright points (say $B_{1}$ and $B_{2}$ ) exhibit a certain amount of coherence. To examine this further, we worked out the correlation coefficients for a number of pairs of bright points using the digital data. We have also computed the correlation coefficients by displacing the data chain of one bright point against the other (up to $5 \mathrm{steps}$ ) in steps of $12 \mathrm{~s}$ which is the digitizing interval. These are presented in Table II. It is seen that there is a suggestion of a weak correlation (ranging from 20\% to 40\%) between the wave trains of two bright points that are within a distance of 8-10 arc sec, the exceptions being $B_{16}$ and $B_{22}, B_{25}$ and $B_{17}$, $B_{29}$ and $B_{23}$. But there is no case of a bright point pair where the correlation is $20 \%$ or above when the distance between them is 10 arc sec or more. These results taken by themselves cannot support unambiguously the existence of a coherence over a distance of 8-10 arc sec, which is reminiscent of the mesogranular structure at the photospheric levels (Sivaraman, 1973; November et al., 1981). But taken along with the evidence provided by Dame and Martic (1987) for the existence of such cells derived from the coherent behaviour of bright points in a long sequence of $\mathrm{K}$-line filtergrams this suggests that cells similar to the mesogranular cells possibly exist at these levels in the chromosphere.

\subsection{CHROMOSPHERIC HEATING}

To compute the energy delivered by the main pulse we evaluated the excess flux in the $1 \AA$ pass band when $H_{2 V}$ is at its peak brightness (Figure 13) over the quiescent profile using the Labs and Neckel (1968) flux values. The values of the energy flux so obtained from several profiles are tabulated in Table III

To estimate the energy delivered by all the bright points over the entire visible hemisphere of the Sun, we made counts of the bright points over a large number of network elements from the excellent $K_{3}$ spectroheliogram obtained at the McMath telescope of the Kitt Peak National Observatory by Bruce Gillespie. We find that on average there are $12-20$ bright points within a network cell. But this, being a spectroheliogram in $K_{3}$, would not show all the bright points that can be seen in a $K_{2 V}$ spectroheliogram and thus would always represent a lower limit of their total numbers. The impression one gets from Figure 1 is that in a quiet region, over $80 \%$ of the length of the slit is occupied by the bright points at the $H_{2} \mathrm{~V}$ wavelengths, and so the counts from even the best spectroheliogram available would be an underestimation of their total number.

Taking the mean energy per bright point as $10^{9} \mathrm{ergs} \mathrm{cm}^{-2} \mathrm{~s}^{-1}$ and its average diameter as 1.5 arc sec and the total number over the entire solar surface as $5 \times 10^{4}$, the energy radiated at the sites of all the bright points turns out to be 
TABLE II

Phase coherence and the correlation coefficients of the bright points

\begin{tabular}{|c|c|c|c|c|c|c|c|}
\hline \multirow[t]{3}{*}{ Bright points } & \multirow{3}{*}{$\begin{array}{l}\text { Distance between } \\
\text { them } \\
\text { in arc sec }\end{array}$} & \multicolumn{6}{|c|}{ Correlation coefficient $(r)$} \\
\hline & & \multicolumn{6}{|c|}{ Phase shift, $\Delta t(\mathrm{~s})$} \\
\hline & & 0 & 12 & 24 & 36 & 48 & 60 \\
\hline$B_{22}$ and $B_{28}$ & 200.00 & 0.10 & 0.20 & 0.39 & 0.30 & 0.20 & 0.10 \\
\hline$B_{17}$ and $B_{19}$ & 76.0 & 0.1 & 0.15 & 0.30 & 0.28 & 0.35 & 0.20 \\
\hline$B_{3}$ and $B_{5}$ & 20.85 & 0.10 & 0.11 & 0.46 & 0.25 & 0.15 & 0.09 \\
\hline$B_{1}$ and $B_{2}$ & 3.00 & 0.25 & 0.45 & 0.25 & 0.28 & 0.20 & 0.30 \\
\hline$B_{12}$ and $B_{13}$ & 3.05 & 0.60 & 0.10 & 0.25 & 0.10 & 0.15 & 0.10 \\
\hline$B_{12}$ and $B_{14}$ & 7.10 & 0.25 & 0.12 & 0.15 & 0.45 & 0.20 & 0.20 \\
\hline$B_{13}$ and $B_{14}$ & 3.05 & 0.22 & 0.20 & 0.35 & 0.10 & 0.10 & 0.20 \\
\hline$B_{10}$ and $B_{11}$ & 5.70 & 0.20 & 0.32 & 0.45 & 0.28 & 0.21 & 0.15 \\
\hline$B_{9}$ and $B_{10}$ & 3.70 & 0.18 & 0.20 & 0.35 & 0.30 & 0.20 & 0.18 \\
\hline$B_{8}$ and $B_{9}$ & 3.00 & 0.20 & 0.38 & 0.30 & 0.22 & 0.18 & 0.10 \\
\hline$B_{12}$ and $B_{21}^{a}$ & 10.00 & 0.11 & 0.17 & 0.23 & 0.16 & 0.12 & 0.09 \\
\hline$B_{15}$ and $B_{16}{ }^{\mathrm{a}}$ & 2.86 & 0.41 & 0.55 & 0.35 & 0.28 & 0.20 & 0.13 \\
\hline$B_{16}$ and $B_{22}{ }^{a}$ & 2.86 & 0.15 & 0.22 & 0.40 & 0.38 & 0.26 & 0.18 \\
\hline$B_{22}$ and $B_{29}{ }^{a}$ & 11.44 & 0.11 & 0.18 & 0.22 & 0.35 & 0.48 & 0.60 \\
\hline$B_{24}$ and $B_{32}{ }^{\mathrm{a}}$ & 2.50 & 0.18 & 0.21 & 0.38 & 0.27 & 0.22 & 0.15 \\
\hline$B_{25}$ and $B_{17^{a}}$ & 2.14 & 0.08 & 0.12 & 0.13 & 0.20 & 0.26 & 0.28 \\
\hline$B_{27}$ and $B_{28}^{\mathrm{a}}$ & 14.30 & 0.10 & 0.11 & 0.13 & 0.20 & 0.38 & 0.25 \\
\hline$B_{29}$ and $B_{23}{ }^{\mathrm{a}}$ & 4.30 & 0.10 & 0.15 & 0.18 & 0.30 & 0.50 & 0.35 \\
\hline$B_{30}$ and $B_{31}$ & 2.50 & 0.18 & 0.21 & 0.38 & 0.27 & 0.22 & 0.15 \\
\hline
\end{tabular}

${ }^{a}$ The $H_{2 V}$ plots of these bright points combination are not displayed here but the digitized values are used for calculating the correlation coefficients.

$15.2 \times 10^{6} \mathrm{ergs} \mathrm{cm}^{-2} \mathrm{~s}^{-1}$. Even if we assume that only $10 \%$ of this energy is available at any one time, we have the total energy radiated from the bright points $=15.2 \times 10^{5} \mathrm{ergs} \mathrm{cm}^{-2} \mathrm{~s}^{-1}$. Assuming a similar value for the $\mathrm{K}$ line, the radiation from the $\mathrm{H}$ and $\mathrm{K}$ lines from the bright points alone would be $30.4 \times 10^{5}$ ergs $\mathrm{cm}^{-2} \mathrm{~s}^{-1}$. The energy radiated by the calcium ions is $3.8 \times 10^{6} \mathrm{~cm}^{-2} \mathrm{~s}^{-1}$ according to the Anderson and Athay model (1989). It should be borne in mind that their model is for an average chromosphere and not specifically for the inner network bright points. We have not computed the radiation loss from the network boundaries. Once this is available it would be possible to estimate the total radiation from the bright points and the network together in the $\mathrm{H}$ and $\mathrm{K}$ lines and compare with model values. The earlier estimates by Liu (1974) and Cram and Dame (1983) attempted to match with the value of model C of VAL81, which itself is only $\frac{1}{3}$ of the estimate of Anderson and Athay (1989). Liu (1974) estimated the excess radiation loss from the bright points based on a very bright structure and therefore 


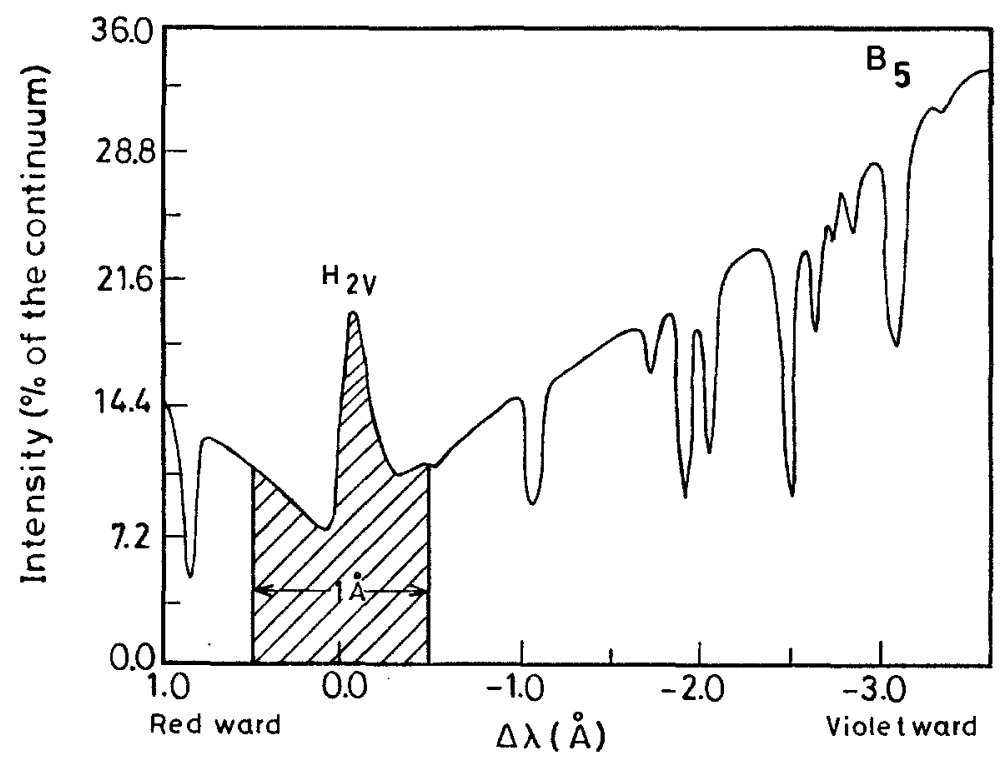

Fig. 13. H-line profile of the bright point $\left(B_{5}\right)$ when the energetic main pulse reached the $H_{2 V}$ level. The hatched region represents the flux in the $1 \AA$ pass band in terms of the continuum brightness. The energy flux contained in the $1 \AA$ pass band ( $1 \AA \mathrm{H}$ index $)$ is the energy excess in the hatched areas over a reference profile.

would seem to represent the very upper limit. From our study (Table III) the energy radiated by the most energetic of the bright points of class I is only 2-3 times that of the most ordinary type of bright point of class III. Thus Liu's estimate can at the most be only 2-3 times the real value and should be taken as a good approximation. Now Cram and Dame (1983) estimated the radiated energy flux in the $\mathrm{H}$ line as $3.9 \times 10^{5} \mathrm{ergs} \mathrm{cm}^{-2} \mathrm{~s}^{-1}$, and considered this a good agreement with the radiative cooling rate by the $\mathrm{H}$ line $\left(5 \times 10^{5} \mathrm{ergs} \mathrm{cm}^{-2} \mathrm{~s}^{-1}\right)$ in the model C of VAL81. Dame, Gouttebroze, and Malherbe (1984) state that Cram and Dame (1983) arrived at this value from the difference between the $\mathrm{H}$ index of the average of the lowest $10 \%$ profiles and the global average. The lowest profiles we have seen pertain to the dark whisker phase. By taking the global average index, they have sought a lower limit for this index, since the global average contains contributions from the bright points, the network boundaries, as well as the dark regions within the network, thus diluting the contribution from the bright points. Whereas, Liu's (1974) calculation brings out the contribution from the bright points alone, and ours is still better as it takes into acocunt all the varieties of the bright points at the same time without diluting it with contributions from other structures. Cram and Dame (1983) were convinced that the bright points are the principal contributors to the radiation loss when they wrote 'since the observed radiation loss from the cell points is of the same order as the theoretical radiation loss from the quiet chromosphere, the cell points themselves are the sites of a significant fraction of the mechanical energy 
TABLE III

Energy flux in the main pulses of the bright points

\begin{tabular}{|c|c|c|}
\hline Class & Bright point & $\begin{array}{l}\text { Energy flux } \\
\times 10^{9} \mathrm{ergs}^{-2} \mathrm{~s}^{-1}\end{array}$ \\
\hline \multirow[t]{13}{*}{ I } & $B_{1}$ & 2.26 \\
\hline & $B_{2}$ & 1.85 \\
\hline & $B_{5}$ & 2.40 \\
\hline & $B_{17}$ & 1.24 \\
\hline & $B_{19}$ & 2.26 \\
\hline & $B_{20}$ & 1.31 \\
\hline & $B_{22}$ & 2.36 \\
\hline & $B_{25}$ & 1.00 \\
\hline & $B_{27}$ & 1.60 \\
\hline & $B_{28}$ & 2.10 \\
\hline & $B_{29}$ & 1.10 \\
\hline & $B_{31}$ & 0.50 \\
\hline & $B_{32}$ & 0.70 \\
\hline \multirow[t]{9}{*}{ II } & $B_{3}$ & 1.73 \\
\hline & $B_{6}$ & 0.90 \\
\hline & $B_{12}$ & 1.16 \\
\hline & $B_{13}$ & 1.21 \\
\hline & $B_{14}$ & 1.25 \\
\hline & $B_{15}$ & 1.20 \\
\hline & $B_{16}$ & 0.91 \\
\hline & $B_{21}$ & 2.09 \\
\hline & $B_{30}$ & 0.60 \\
\hline \multirow[t]{7}{*}{ III } & $B_{7}$ & 0.41 \\
\hline & $B_{8}$ & 0.45 \\
\hline & $B_{9}$ & 1.22 \\
\hline & $B_{10}$ & 1.19 \\
\hline & $B_{11}$ & 0.49 \\
\hline & $B_{23}$ & 1.42 \\
\hline & $B_{24}$ & 0.91 \\
\hline
\end{tabular}

dissipation responsible for the heating of the quiet, inner network chromosphere'. We would like to remind the reader that the equality of the radiative flux does not establish any causal connections; but it is clear that the bright points are a direct manifestation of the dissipation processes that heat the inner network chromosphere. These are in antithesis to the conclusion by Skumanich, Smythe, and Frazier (1984) that these structures cannot be important contributors to the Kflux. This may be due to an underestimate of the contrast value they used to evaluate 
the contribution from the bright points. Another point in favour of the dominance of the contribution from the bright points is the following: the bright points and the network are the only two known main contributors to the emission in the quiet chromosphere, and between them the bright points are the centres where the 3-min brightness oscillations are seen, whereas the network shows variations far above this value. Since the 3-min oscillations are the strikingly prominent feature at this chromospheric level, the bright points are necessarily the dominant contributors to the heating of the chromosphere.

The chromosphere is highly structured and can be identified with the atmosphere inside the bright points. With their association with the magnetic fields, they can be considered as magnetic flux tubes. The main pulse is a sort of compressional wave that propagates vertically along the flux tube and carries the energy to the chromosphere and provides the energy for its heating. In the case of the class I bright points, the value of the magnetic field strength is high and most likely to be in the range $40-80 \mathrm{G}$ spread over a linear scale size $\sim 2$ arc sec. The observations are seeing-limited and so the fields would definitely be more when the real linear size of a bright point is less ( $\sim 1$ arc sec or even less) as can be seen from the UV spectroheliograms.

Regarding the heating by the 3 -min waves, various possibilities are currently under debate and they fall into two classes: one that invokes magnetic association for the bright points and the others which consider the bright points as a pure hydrodynamic phenomenon without requiring any connection with magnetic fields. According to Kalkofen $(1989,1991 a, b)$ the bright points with their magnetic fields act as individual flux tubes and they can form effective channels for the upward propagation of 3-min waves and thus the bright points mark the sites of chromospheric heating that takes place exclusively in strong-field flux tubes. In the presence of magnetic fields, the cut-off period becomes higher $(>3 \mathrm{~min}$ ) and this removes the severe constraint in the modelling. Opposed to this are models that consider the bright points as hydrodynamic phenomena and explain the heating through shock heating (Rutten and Uitenbroek, 1991; Ulmschneider, 1991) although they differ in detail about how the shocks are formed. In Deubner's (1991) model the energy is induced by the 3 -min oscillations of the $K_{3}$ layer, which are nonlinear and subsonic.

However, none of the models describe fully the bright points and the way the waves near the cut-off period are propagated. Thus the $H_{2 V}$ (or $K_{2 V}$ ) bright points remain as enigmatic as before and will continue to draw the attention of both observers and theoreticians as they seem to hold the key for an understanding of the quiet chromosphere of the Sun and hence also of other Sun-like stars.

\section{Conclusions}

We have used very high quality $\mathrm{H}$-line spectra with spatial resolution of 1 arc sec or better to study the time evolution of the $H_{2 V}$ bright points and the main conclusions 
that have emerged are:

The bright points can be grouped into 3 classes: class I bright points have the most energetic 'main pulse' as seen from the enhancement of $I_{H 2 V}$ at the brightest phase. In all 3 classes the main pulse appears to be the carrier of the non-thermal energy and is followed by many impulses whose amplitudes decrease exponentially. We tentatively suggest that the main pulse is the perturbing disturbance which throws the medium into a resonating mode seen as the follower pulses with a period identical to the exciting pulse. We admit that more observations lasting for longer duration are required to confirm this picture. The number of follower pulses is maximum for class I (8-10) and less for class II and class III (4-6). The average period of all these pulses lies in the range 150-210 s. We suggest that the differences in the evolutionary behaviour of the bright points of the 3 classes are related to the differences in the magnetic fields with which the bright points are associated: class I bright points being associated with the highest field and class III with low fields. Our calculations confirm that the energy radiated by the bright points can account for a substantial fraction of the radiation from the quiet chromosphere.

\section{Acknowledgements}

One of us (KRS) wishes to express with pleasure his grateful thanks to the staff at the VTT, National Solar Observatory, Sacramento Peak, for help while acquiring the observations and to Dr Raymond Smartt, Deputy Director for his keen interest in the course of the analysis and for the warm hospitality he offered him during many short spells of stay at the mountain which made the stay enjoyable and productive. We are thankful to Prof. J. C. Bhattacharyya, Indian Institute of Astrophysics, for the encouragement during the investigation and to $\mathrm{Mr} \mathrm{B}$. A. Varghese for his help in the reduction of the data. We are grateful to Dr W. Kalkofen and to Prof. S. M. Chitre for their valuable comments and to Dr Robert Rutten who as a referee made many valuable suggestions.

\section{References}

Anderson, L. S. and Athay, R. G.: 1989, Astrophys. J. 346, 1010.

Bappu, M. K. V. and Sivaraman, K. R.: 1971, Solar Phys. 17, 316.

Beckers, J. M.: 1964, AFCRL Environmental Research Paper, No. 49.

Beckers, J. M., Mauter, H. A., Mann, G. R., and Brown, D. R.: 1972, Solar Phys. $25,81$.

Cram, L. E.: 1974, Solar Phys. 37, 75.

Cram, L. E. and Dame, L.: 1983, Astrophys. J. 272, 355.

Dame, L.: 1984, in Stephen L. Keil (ed.), Small Scale Dynamical Processes in Quiet Stellar Atmospheres, National Solar Observatory Conference, Sunspot, July 11, 1983, p; 54.

Dame, L. and Martic, M.: 1987, Astrophys. J. 314, L15.

Dame, L., Gouttebroze, P., and Malherbe, J. M.: 1984, Astron. Astrophys. 130, 331.

Deubner, F. L.: 1991, in P. Ulmschneider, E. R. Priest, and R. Rosner (eds.), Mechanisms of Chromospheric and Coronal Heating, p. 6.

Durrant, C. J., Grossmann-Doerth, U., and Kneer, J.: 1976, Astron. Astrophys. 51, 95. 
Hale, G. E. and Ellerman, F.: 1903, Publ. Yerkes Obs. III, (1), 3.

Jensen, E. and Orrall, F. Q.: 1963, Astrophys. J. 138, 252.

Kalkofen, W.: 1989, Astrophys. J. 346, L37.

Kalkofen, W.: 1991a, in P. UImschneider, E. R. Priest, and R. Rosner (eds.), Mechanisms of Chromospheric and Coronal Heating, p. 54.

Kalkofen, W.: 1991b, in A. N. Cox, W. C. Livingston, and M. S. Matthews (eds.), Solar Interior and Atmosphere, University of Arizona Press, Tucson, p. 911.

Kulaczewski, J.: 1992, Astron. Astrophys. 261, 602.

Labs, D. and Neckel, H.: 1968, Z. Astrophys. 69, 1.

Liu, S. Y.: 1974, Astrophys. J. 189, 359.

November, L. J., Toomre, J., Gebbie, K. B., and Simon, G. W.: 1981, Astrophys. J. 245, L123.

Punetha, L. M.: 1974, Bull. Astron. Inst. Czech. 25, 212.

Rutten, R. J. and Uitenbroek, H.: 1991, Solar Phys. 134, 15.

Sivaraman, K. R.: 1973, Solar Phys. 33, 319.

Sivaraman, K. R. and Livingston, W. C.: 1982, Solar Phys. 80, 227.

Skumanich, A., Smythe, C., and Frazier, E. N.: 1975, Astrophys. J. $200,747$.

Ulmschneider, P.: 1991, in P. Ulmschneider, E. R. Priest, and R. Rosner (eds.), Mechanisms of Chromospheric and Coronal Heating, p. 328.

Vernazza, J. E., Avrett, E. H., and Loeser, R.: 1981, Astrophys. J. Suppl. Ser. 45, 635.

White, O. R. and Suemoto, Z.: 1968, Solar Phys. 3, 523.

Zirin, H.: 1974, Solar Phys. 38, 91. 